\title{
Prediction of blunt traumatic injuries and hospital admission based on history and physical exam
}

\author{
Alan L. Beal ${ }^{*}$, Mark N. Ahrendt ${ }^{3}$, Eric D. Irwin³ ${ }^{3}$ John W. Lyng ${ }^{3}$, Steven V. Turner², Christopher A. Beal²,
} Matthew T. Byrnes ${ }^{3}$ and Greg A. Beilman ${ }^{2}$

\begin{abstract}
Background: We evaluated the ability of experienced trauma surgeons to accurately predict specific blunt injuries, as well as patient disposition from the emergency department (ED), based only on the initial clinical evaluation and prior to any imaging studies. It would be hypothesized that experienced trauma surgeons' initial clinical evaluation is accurate for excluding life-threatening blunt injuries and for appropriate admission triage decisions.

Methods: Using only their history and physical exam, and prior to any imaging studies, three (3) experienced trauma surgeons, with a combined Level 1 trauma experience of over 50 years, predicted injuries in patients with an initial GCS (Glasgow Coma Score) of 14-15. Additionally, ED disposition (ICU, floor, discharge to home) was also predicted. These predictions were compared to actual patient dispositions and to blunt injuries documented at discharge.
\end{abstract}

Results: A total of 101 patients with 92 blunt injuries were studied. 43/92 (46.7\%) injuries would have been missed by only performing an initial history and physical exam ("Missed injury"). A change in treatment, though often minor, was required in 19/43 (44.2\%) of the missed injuries. Only 1/43 (2.3\%) of these "missed injuries" (blunt aortic injury) required surgery. Sensitivity, specificity, and accuracy for injury prediction were 53.2, 95.9, and $92.3 \%$ respectively. Positive and negative predictive values were 53.8 and $95.8 \%$ respectively. Prediction of disposition from the ED was 77. $8 \%$ accurate. In 7/34 (20.6\%) patients, missed injuries led to changes in disposition. "Undertriage" occurred in 9/99 (9.1\%) patients (Predicted for floor but admitted to ICU). Additionally, 8/84 (9.5\%) patients predicted for floor admission were sent home from the ED; and 5/13 (38.5\%) patients predicted for ICU admission were actually sent to the floor after complete evaluations, giving an "overtriage" rate of 13/99 (13.1\%) patients.

Conclusions: In a neurologically-intact group of trauma patients, experienced trauma surgeons would have missed 46. $7 \%$ of the actual injuries, based only on their history and physical exam. Once accurate diagnoses of injuries were completed, usually with the help of CT scans, admission dispositions changed in $20.6 \%$ of patients. Treatment changes occurred in $44.2 \%$ of the missed injuries, though usually minimal. Broad elimination of early imaging studies in alert, blunt trauma patients cannot be advocated.

Keywords: Trauma, Injuries, Triage, Imaging

Abbreviations: BAL, Blood alcohol level; CT, Computed tomography; CXR, Chest X-ray; EAST, Eastern association for the surgery of trauma; ED, Emergency Department; GCS, Glascow coma score; ICU, Intensive care unit; ISS, Injury severity score; NEXUS, National emergency radiologic utilization study; TRAINS, Traumatic aortic injury score

\footnotetext{
* Correspondence: Alan.beal@northmemorial.com

${ }^{1}$ North Memorial Medical Center, 3300 Oakdale Ave N, Robbinsdale, MN

55431, USA

Full list of author information is available at the end of the article
} 


\section{Background}

Diagnostic accuracy and efficiency are important in the initial trauma evaluation. Goals also include limitation of patients' time spent in the ED, compiling an accurate list of injuries, and making rapid, safe, disposition decisions; i.e.; operating room, ICU, floor or discharge to home. Early and aggressive imaging of the trauma patient, using plain films, ultrasound, and a wide variety of CT scans has become commonplace in the trauma evaluation. The yield of CT scans varies in blunt trauma victims, creating inconsistent recommendations for their use, especially in alert patients [1-3]. We returned to the basics of clinical medicine in this prospective study and evaluated the accuracy of the history and physical exam when carried out by experienced trauma surgeons on a group of awake patients. We also tried to predict the emergency department disposition of these patients, hoping to speed up their admission process.

\section{Methods and study design}

The study was reviewed by the North Memorial IRB, and waiver of consent requirements was granted. The study was conducted by three trauma surgeons with similar levels of training and experience. All surgeons completed surgical residencies between 1988 and 1994, and have a combined experience of 56 years at our Level 1 trauma center. A total of 101 non-consecutive blunt trauma patients with a Glasgow Coma Score of 14-15 were evaluated over nine (9) months, prior to completion of any radiologic imaging. Specific injuries were predicted, based only on the history and physical exam. Patients were excluded if any imaging studies, including ultrasonography, had been completed prior to the trauma surgeons' evaluation. Patients underwent collection of medical history and a physical examination, and specific injuries were then predicted in each of eleven (11) categories, using a standardized prediction worksheet. The patient's emergency department disposition was also predicted and recorded prior to imaging studies; i.e., ICU, floor, or discharge to home. "Missed injuries" were defined as those not predicted by the trauma surgeon on the admission prediction worksheet, but eventually diagnosed during the hospitalization. Most patients had multiple imaging studies after predictions had been made. All missed injuries, incorrect diagnoses and incorrect patient dispositions were recorded. Any change in treatment plans were noted for each of the missed injuries.

Sensitivity, specificity, accuracy, as well as positive and negative predictive values was determined for all predicted injuries. The overall accuracy, "overtriage" and "undertriage" rates were determined for the predicted dispositions to the ICU, floor or discharge to home. A comparison of patients' ages and ISS was made between the group with "missed injuries" and those without missed injuries, using a paired $t$ test method. Those patients with a GCS of 14 were compared in the same two groups ("Missed injury vs no missed injury) with those with GCS of 15, using a two-tailed Fisher's exact test.

\section{Results}

Table 1 gives a breakdown of the number of patients seen and entered into the study by each surgeon, as well as the number of injuries diagnosed and "missed" by each of them. Table 2 gives basic demographics of the 101 blunt trauma patients.

Table 3 gives details of the three combined surgeons' accuracies in each of the eleven injury categories addressed on the prediction worksheet.

Combined, the surgeons' overall outcome in predicting injuries based on the initial history and physical exam includes a 53.2 sensitivity, 95.9 specificity, 95.8 negative predictive value, and a $53.8 \%$ positive predictive value, for an overall accuracy of $92.3 \%$.

Results of the predictions for the disposition of the patients are shown in Table 4. A total of 9/84 (10.7\%) patients predicted for a floor admission were instead admitted to the ICU due to their missed injuries ("undertriage"). Also, 8/84 (9.5\%) patients predicted to go to the floor were actually able to go home after their evaluations, while $5 / 13$ (38.5\%) predicted to go to the ICU were able to go to the floor, for an "overtriage" rate of 13/97 (13.4\%). Overall disposition accuracy was $77.8 \%$.

A total of 43 "missed injuries" occurred in 34 patients. Nine patients each had two missed injuries. The patients with missed injuries had a range of ages from 11-88. The mean age of those patients with missed injuries was older than those with accurate diagnoses (42.8 vs $34.5 ; p<0.03)$ There were only two missed injuries in the eight pediatric patients, a clavicle fracture in an 11 year old, and a minimal wrist fracture in a 14 year old.

A total of $6 / 34$ (17.6\%) patients with missed injuries had a GCS of 14, while 4/67 (6.0 \%) without missed injuries had GCS of 14. ( $p=0.08)$ The mean injury severity score (ISS) of the group with missed injuries was 12.6, as compared to an ISS of 5.7 in those patients without missed injuries. $(p<0.0001)$ Surprisingly, only $4 / 34$ (11.8\%) with missed injuries had elevated blood alcohol levels (BAL) at the time of the initial evaluation.

Table 1 Injuries and missed injuries

\begin{tabular}{lllll}
\hline Surgeon & $\begin{array}{l}\text { \# of } \\
\text { Pts }\end{array}$ & $\begin{array}{l}\text { Total \# } \\
\text { of injuries }\end{array}$ & $\begin{array}{l}\text { \# of Pts } \\
\text { with no injuries }\end{array}$ & $\begin{array}{l}\text { \# of missed } \\
\text { injuries }\end{array}$ \\
\hline Surgeon A & 18 & 17 & 7 & 9 \\
Surgeon B & 45 & 46 & 16 & 15 \\
Surgeon C & 38 & 29 & 15 & 19 \\
Total & 101 & 92 & 38 & 43 \\
\hline
\end{tabular}


Table 2 Patient Demographics

\begin{tabular}{ll}
\hline Demographics & Data \\
\hline Sex & Males: $N=70$ Females: $N=31$ \\
Age & Mean: 38.1 Range: $3-88$ \\
ISS & Mean: 8.0 Range: $0-38$ \\
GCS Score & GCS of 15: $N=91$ GCS of $14: N=10$ \\
\hline
\end{tabular}

Overall, 22/97 (22.7 \%) patients had a change in their disposition compared to that predicted. Two patients did not have predictions made at the initial evaluation. Change in admission disposition due to at least one missed injury occurred in $7 / 34$ (20.6 \%) patients.. Change in therapy occurred in 19/43 (44.2\%) missed injuries, though most of these treatment changes were modest; e.g., extremity splinting, frequent neurological exam; analgesia. Only one patient required surgery for an injury not predicted by the initial history and physical exam: a blunt thoracic aortic injury.

Table 5 lists the number of missed injuries by categories and whether there were changes in treatment of disposition based on missed injuries.

Only $1 / 6$ of the missed traumatic brain injuries would be considered significant. None of the cervical spine fractures were considered serious, with only one needing a cervical collar. Two of 3 liver injuries were not predicted and both of these patients had short ICU admissions and successful non-operative management. Both of the "missed" pelvic fractures were minor. The surgeons did not predict $8 / 12$ of the thoracolumbar fractures, but none of these fractures required surgery, and only 2 required orthotics. All of the "missed" extremity, clavicular and scapular fractures were modest; none required surgery. Both of the vascular injuries were not suspected on the history and physical. The unilateral vertebral artery injury
Table 4 Prediction of Patient Disposition

\begin{tabular}{llll}
\hline $\begin{array}{l}\text { Predicted } \\
\text { disposition }\end{array}$ & $\begin{array}{l}\text { Actual disposition: } \\
\text { floor }\end{array}$ & $\begin{array}{l}\text { Actual disposition: } \\
\text { ICU }\end{array}$ & $\begin{array}{l}\text { Actual disposition: } \\
\text { home }\end{array}$ \\
\hline Floor & 67 & 9 & 8 \\
ICU & 5 & 8 & 0 \\
Home & 0 & 0 & 2 \\
\hline
\end{tabular}

was treated with anti-platelet therapy. The blunt thoracic aortic injury required an endovascular stent graft.

\section{Discussion}

The history and physical exam can serve an important role in most trauma work-ups. Advanced Trauma and Life Support (ATLS) programs emphasize the use of a history and exam during both the primary and secondary surveys. Treatment of suspected life-threatening injuries can occur based only on the physical exam [4].

For this study, we challenged our most experienced trauma surgeons to prospectively predict injuries, as well as the patients' emergency department (ED) disposition, prior to any imaging studies being completed. Evaluating a group of alert trauma patients (GCS 14-15) and knowing the accuracy of our predictions is a first step in potentially reducing the number of imaging studies, while decreasing patient time spent in the ED. In our study, however, 43/92 (46.7 \%) injuries would have been missed if only the history and exam had been used for initial definitive diagnoses ("Missed injury").

The reasons for our high missed injury rate are not clear. By choosing our most experienced surgeons, the impact of the inexperience factor was reduced. Complacence or inattention to detail in the history and physicals may have occurred despite their significant experience. In our institution, the trauma surgeons do not have regular

Table 3 Accuracy of Predicting Injuries with $\mathrm{H} / \mathrm{P}$

\begin{tabular}{|c|c|c|c|c|c|c|c|c|c|}
\hline Injury & $\mathrm{TP}^{1}$ & $F P^{2}$ & $\mathrm{TN}^{3}$ & $\mathrm{FN}^{4}$ & Sens $^{5}$ & $\operatorname{Spec}^{6}$ & $\mathrm{NPV}^{7}$ & $\mathrm{PPV}^{8}$ & $\overline{\text { Accur }^{9}}$ \\
\hline Brain & 3 & 6 & 86 & 6 & 33.3 & 93.5 & 93.5 & 33.3 & 88.1 \\
\hline Cervical Fracture & 1 & 4 & 92 & 4 & 20.0 & 95.8 & 95.8 & 20.0 & 92.1 \\
\hline Rib Fractures & 7 & 9 & 78 & 7 & 50.0 & 89.7 & 91.8 & 43.8 & 84.2 \\
\hline Pneumothorax & 1 & 1 & 96 & 3 & 25.0 & 99.0 & 97.0 & 50.0 & 96.0 \\
\hline Solid organ injury & 1 & 4 & 94 & 2 & 33.3 & 95.9 & 97.9 & 20.0 & 94.1 \\
\hline Pelvic Fracture & 4 & 4 & 91 & 2 & 66.7 & 97.8 & 97.8 & 50.0 & 94.1 \\
\hline T/L Spine Fracture & 3 & 4 & 85 & 8 & 27.2 & 95.5 & 91.4 & 42.9 & 87.1 \\
\hline Extremity Fracture & 22 & 6 & 68 & 5 & 81.5 & 91.9 & 93.2 & 78.6 & 89.1 \\
\hline Clavicle Fracture & 5 & 1 & 91 & 4 & 55.6 & 98.9 & 95.8 & 83.3 & 95.0 \\
\hline Vascular Injury & 0 & 0 & 99 & 2 & 0 & 100 & 98.0 & 0 & 98.0 \\
\hline Spinal Cord Injury & 2 & 3 & 96 & 0 & 100 & 96.7 & 100 & 40.0 & 97.0 \\
\hline Total & 49 & 42 & 976 & 43 & 53.2 & 95.9 & 95.8 & 53.8 & 92.3 \\
\hline
\end{tabular}

${ }^{1} T P$ True positive, ${ }^{2} F P$ False positive, ${ }^{3} T N$ True negative, ${ }^{4} F N$ False negative, ${ }^{5}$ Sens Sensitivity, ${ }^{6}$ Spec Specificity, ${ }^{7} N P V$ Negative predictive value, ${ }^{8} P P V$ Positive predictive value, ${ }^{9}$ Accur Accuracy 
Table 5 Numbers of "Missed Injuries" by Type

\begin{tabular}{llll}
\hline Injury type & $N=43$ & $\begin{array}{l}+ \text { Change } \\
\mathrm{Rx}\end{array}$ & $\begin{array}{l}+ \text { Change } \\
\text { disposition }\end{array}$ \\
\hline Traumatic Brain Injuries & 6 & 3 & 3 \\
Cervical Spine Fractures & 4 & 1 & 0 \\
Ribs/Sternum & 7 & 4 & 1 \\
Pneumothoraces & 3 & 0 & 1 \\
Solid Organ Injuries & 2 & 1 & 0 \\
Pelvic Fractures & 2 & 0 & 0 \\
Spine Fractures & 8 & 2 & 0 \\
Extremity Fractures & 5 & 4 & 0 \\
Clavicle/Scapular Fractures & 4 & 2 & 0 \\
Vascular & 2 & 2 & 2 \\
\hline
\end{tabular}

house staff support and remain the frontline decisionmakers for our trauma patients. In the modern trauma evaluation, ongoing reliance on imaging studies, such as CT scans, certainly could lead to less focus and concentration on the history and physical exam and erode these clinical skills.

We also identified poor "under/over"-triage rates when relying on the history and physical exam. Since our injury prediction accuracy was unacceptably low, this was likely the major cause for the undertriage rate for patient dispositions.

The injury severity score (ISS) was higher in the 34 patients with missed injuries (12.6 vs $5.7 ; p<.0001)$. This suggests that the unidentified injuries, or the associated pain, could have been a confounding factor in accurately assessing the extent of injury. The ISS is determined retrospectively by the trauma registrars and thus would not have changed based on our definition of a "missed injury".

The Glasgow Coma Score (GCS) of the two groups (missed injuries vs. no missed injuries) was not significantly different (14.8 vs 14.7). However, 6/34 (17.6 \%) patients with missed injuries had a GCS of 14 , while only $2 / 67$ (3.0 \%) patients without missed injuries had a GCS of 14 . $(p=0.08)$. Though not significant, the trend here between differences in mental status could have played a role in the overall accuracy of our history and physical exams.

The mean age of those patients with "missed injuries" was older than those with accurate diagnoses (42.8 vs 34.5: $p<0.03)$. It is not unusual for elderly patients to have abnormal pain thresholds in a variety of clinical scenarios. This could have had an effect on the history and physical exams of our patients.

It did not appear that alcohol played an important role in our diagnoses of injuries. A total of 14/50 (28\%) patients in the group without missed injuries had elevated blood alcohol levels (BAL). Only 4/35 (11.4\%) of patients in the group with "missed injuries" had elevated $\operatorname{BAL}(p=0.10)$.
The study was originally designed to have a wider range of clinicians (medical students, surgical interns and critical care fellows) also make predictions in order to gather data from clinicians with a more varied range experiences however their rotations were relatively short and the number of evaluations completed by these clinicians was very small. Given the limited number of surgeons' experiences within this study, it is possible that the accuracy of the predictions cannot be extrapolated to other trauma centers or surgeons. Results from this study indicate that a full evaluation, including imaging, in trauma patients will provide the most beneficial care plan, however further investigations are required to confirm these findings.

A number of studies have addressed the diagnostic accuracy of the history/physical in a variety of traumatic injuries. None have used a small, consistent and experienced group of clinicians, as we did in this study. Many have employed retrospective chart reviews, rather than a prospective approach.

Hoping to reduce the number of CT scans in blunt trauma patients, Tillou, Cryer and colleagues would have missed almost $17 \%$ of injuries with use of their initial clinical evaluation [5]. Even in awake patients with a normal exam and stable hemodynamics, Salim et al. found "clinically significant findings" in $3.5 \%$ of head CT's, $5.1 \%$ of cervical CT's, $7.1 \%$ of abdominal CT's and $19.6 \%$ of chest CT scans. These findings changed patient management in $19 \%$ of the patients [6].

Previous studies of traumatic brain injuries report up to a $20 \%$ rate of abnormal head CT's and a $5 \%$ need for craniotomy, even with a normal clinical exam [7-9]. "The Canadian head CT rule" and the "New Orleans criteria" remain the best predictors of clinically-significant brain injuries in alert patients [10-12].

Clinical criteria to rule out cervical spine injuries have been evaluated. The National Emergency Radiologic Utilization Study (NEXUS) included over 34,000 patients in 21 centers, while the "Canadian $\mathrm{C}$ spine rule" prospectively developed clinical criteria to accurately rule out cervical injuries $[13,14]$. These studies were the foundation for other more recent recommendations to help reduce the number of imaging studies needed, while simplifying the cervical evaluations in both adults and children [15-19].

The history and exam can be quite accurate for diagnoses in blunt chest injuries in both adults and children, arguing for fewer imaging studies [20-24]. Blunt abdominal trauma diagnoses can be challenging using only the history/physical. Patients with subjective symptoms and positive physical findings, such as bruising and tenderness, will have intra-abdominal injuries in only about $20 \%$ of cases $[25,26]$. On the other hand, the incidence of actual injuries with a negative exam is also reported to be up to 
$20 \%[4,25-32]$. Other factors such as distracting injuries, low GCS and alcohol intoxication can affect the accuracy of the physical exam [26, 30,33]. Physical exam in children has been shown to be more sensitive, but is still challenging without the support of other modalities such as ultrasound [34, 35].

Bedside clinical assessment for pelvic fractures can be sensitive in the alert patient [36]. False negative exams are present in 1-7 \% of patients with the appropriate mechanism of injury. Physical exam can sometimes be more sensitive than plain $\mathrm{x}$-rays $[37,38]$.

An in-depth review of the accuracy of the history/ physical in diagnoses of thoracolumbar fractures found conflicting results [39]. Several reports support the premise that the lack of symptoms and tenderness predicts a very low risk for fractures [40, 41]. A prospective, predictive study by Holmes and colleagues supports these findings in alert patients [42]. Diagnostic guidelines for thoracolumbar spine evaluations have been established by the Eastern Association for Surgery of Trauma (EAST) [43]. On the other hand, 20-50\% of these fractures have been reported to have no symptoms or physical findings, even in alert patients [44-46].

Musculoskeletal injuries historically have been the most commonly missed traumatic injuries [47, 48]. The incidence of missed injuries or delayed diagnoses of musculoskeletal trauma has been reported to be from 1.3-39\%, with the higher rates seen in the more severely injured, and especially in those with altered mental status [48-51]. More than $20 \%$ of these missed injuries can be clinically significant [52]. The usefulness of the clinical exam in diagnosis of musculoskeletal trauma has not been widely studied, and available data are mostly from studies of low energy, isolated injuries, often seen in ambulatory patients; e.g., elbow, wrist, hand [53-56].

While a great deal has been written about the evaluation and management of penetrating vascular injuries, blunt vascular trauma has been less well-studied. Blunt arterial injuries comprise only about $20 \%$ of arterial trauma and can present with minimal clinical findings [57]. The mechanism or pattern of injury may be the only factor to make one suspicious for arterial injury. Blunt thoracic aortic injuries rarely have a blood pressure differential between the arms and legs. Even CXR's are normal in 7.3-23 \% of those with blunt thoracic aortic injuries [57]. A "traumatic aortic injury score" (TRAINS) has been reported, but relies more on the chest $\mathrm{x}$-ray and the diagnosis of other associated injuries, rather than on the history and exam [58].

Clinical risk factors for a blunt carotid or vertebral artery injury were recently reported in a Western Trauma Association critical decision paper [59]. Unfortunately, up to $20 \%$ of patients with such injuries have none of these risk factors [60]. Emphasizing the importance of timely and accurate diagnoses, the EAST group published practice management guidelines for blunt cerebrovascular injuries, and cite an $80 \%$ morbidity and $40 \%$ mortality rate if neurologic symptoms develop from these injuries [61].

\section{Conclusion}

If only a history and physical exam is used for diagnosis in an alert group of trauma patients (GCS 14-15), experienced trauma surgeons at our hospital missed $46.7 \%$ of their injuries. The reasons for these inaccurate clinical predictions are not clear, though the average injury severity score was higher and the age of the patients greater in those with missed injuries. This same approach to predicting a trauma patient's hospital disposition was $77.8 \%$ accurate, with $9.1 \%$ of patients being "undertriaged" to the floor. Due to these results, though many of the "missed" injuries were minor and often did not require a change in treatment, we cannot advocate a broad elimination of early imaging studies, even in alert trauma patients.

\section{Acknowledgments \\ Not applicable.}

\section{Availability of data and materials}

The data supporting the research findings in this article will not be shared in due to the confidentiality requirements of the participating institutions.

\section{Authors' contributions}

ALB conceived the project, and participated in the development of the study design, data acquisition, and writing of the manuscript. EDI, MNA, MTB and GAB contributed to study design, and also participated in data acquisition and interpretation. SVT and CAB participated in data analysis and interpretation of the data. ALB, GAB and JWL contributed to the critical review and editing of the manuscript. All authors read and approved of the final manuscript.

\section{Competing interests}

The authors declare that they have no competing interests.

Consent for publication

Not applicable.

\section{Ethics approval and consent to participate}

This study was reviewed by the IRB/Ethics Committee at North Memorial Medical Center. The IRB waived the need for informed consent since the study did not involve additional assessments beyond standard of care, and patients were not exposed to any additional risks.

\section{Author details}

${ }^{1}$ North Memorial Medical Center, 3300 Oakdale Ave N, Robbinsdale, MN 55431 , USA. ${ }^{2}$ University of Minnesota, Minnesota, USA. ${ }^{3}$ North Memorial Medical Center, Minnesota, USA.

Received: 21 May 2016 Accepted: 6 August 2016

Published online: 31 August 2016

References

1. Sampson MA, Colquhoun KB, Hennessy NL. Computed tomography whole body imaging in multi-trauma: 7 years experience;". Clin Radiol. 2006;61:365-9.

2. Millo NZ, Plewes C, Rowe BH, Low G. Appropriateness of CT of the chest, abdomen, and pelvis in motorized blunt force trauma patients without signs of significant injury. AJR Am J Roentgenol. 2011;197:1393-8.

3. Milia D, Brasel K. Current use of $C T$ in the evaluation and management of injured patients. Surg Clinics North Am. 2011;91:233. 
4. American College of Surgeons. Advanced Trauma Life Support Student Manual-9th Ed. Chicago: American College of Surgeons; 2012.

5. Tillou A, Cryer HM, et al. Is the use of pan-computed tomography for blunt trauma justified? A prospective evaluation. J Trauma. 2009;67(4):779-87.

6. Salim A, Demetriades $D$, et al. Whole body imaging in blunt multisystem trauma patients without obvious signs of injury: Results of a prospective study. Arch Surg. 2006;141:468-75.

7. Harnan SE, Goodacre SW, et al. Clinical decision rules for adults with minor head injury: a systematic review. J Trauma. 2011;71:245-51.

8. Stein SC, Ross SE. The value of computed tomographic scans in patients with low-risk head injuries. Neurosurgery. 1990;26:638-40.

9. Shackford SR, et al. The clinical utility of computed tomographic scanning, and neurologic examination in the management of patients with minor head injuries. J Trauma. 1992;33:385-94.

10. Stiell IG, CCC Study Group, et al. The Canadian CT head rule for patients with minor head injury. Lancet. 2001;357:1391-96.

11. Stiell IG, Wells GA, et al. Comparison of the Canadian $C T$ head rule and the New Orleans criteria in patients with minor head injury. JAMA. 2005;294:1511-18.

12. Haydel MJ, DeBlieux PM. Indications for computed tomography in patients with minor head injury. N Engl J Med. 2000;343:100-5.

13. Hoffman JR, NEXUS Group, et al. Validity of a set of clinical criteria to rule out injury to the cervical spine in patients with blunt trauma. N Engl I Med. 2000:343:94-9.

14. Stiell IG, et al. The Canadian C-spine rule for radiography in alert and stable trauma patients. JAMA. 2001;286:1841-48.

15. Stiell IG, Wells DA, et al. The Canadian C-spine rule versus the NEXUS lowrisk criteria in patients with trauma. N Engl J Med. 2003;349:2510-8.

16. Stiell IG, Clement CM, et al. Implementation of the Canadian C-spine rule: prospective 12 center cluster randomized trial. BMJ. 2009;339:b4146.

17. Sanchez B, Waxman $K$, et al. Cenvical spine clearance in blunt trauma: Evaluation of a computed tomography-based protocol. J Trauma. 2005;59:179-83.

18. Gonzalez RP, Rodning CB, et al. Clincal examination in complement with computed tomography scan: an effective method for identification of cervical spine injury. J Trauma. 2009;67:1297-304.

19. Viccellio P, Simon H, NEXUS Group, et al. A prospective multicenter study of cervical spine injury in children. Pediatrics. 2001;108:E20.

20. Brink M, Blickman JG, et al. Predictors of abnormal chest $C T$ after blunt trauma: a critical appraisal of the literature. Clin Radiol. 2009:64:272-83.

21. Bokhari F, Barrett J, et al. Prospective evaluation of the sensitivity of physical examination in chest trauma. J Trauma. 2002:53:1135-8.

22. Rodriguez RM, Bjoring A, et al. A pilot study to derive clinical variables for selective chest radiography in blunt trauma patients. Ann Emerg Med. 2006;:47:415-8.

23. Paydar $\mathrm{P}$, Sharifian $\mathrm{M}$, et al. The role of routine chest radiography in initial evaluation of stable blunt trauma patients. Am J Emerg Med. 2012;30:1-4.

24. Holmes JF, Kuppermann N, et al. A clinical decision rule for identifying children with thoracic injuries after blunt torso trauma. Ann Emerg Med. 2002;39:492-9

25. Livingston DH, Malangoni MA, et al. Admission or observation is not necessary after a negative abdominal computed tomographic scan in patients with suspected blunt abdominal trauma: results of a prospective, multi-institutional trial. J Trauma. 1998:44:273-80.

26. Rodriguez $\mathrm{A}$, Shatney $\mathrm{CH}$, et al. Recognition of intra-abdominal injury in blunt trauma victims. A prospective study comparing physical examination with peritoneal lavage. Am Surg. 1982:48:457-9.

27. Nishijima DK, Holmes JF, et al. Does this adult patient have a blunt intraabdominal injury?'. JAMA. 2012;307:1517-27.

28. Poletti PA, Mermillod B, et al. Blunt abdominal trauma patients: Can organ injury be excluded without performing computed tomography? J Trauma. 2004;57:1072-81.

29. Schauer BA, Holmes JF, et al. Is definitive abdominal evaluation required in blunt trauma victims undergoing urgent extra-abdominal surgery? Acad Emerg Med. 2005;12:707-11.

30. Ferrera $P C$, Salluzzo RF, et al. Injuries distracting from intra-abdominal injuries after blunt trauma. Am J Emerg Med. 1998;16:145-9.

31. Holmes JF, Kupperman N, et al. Clinical prediction rules for identifying adults at very low risk for intra-abdominal injuries after blunt trauma. Ann Emerg Med. 2009;54:575-84.

32. Richards JR, Derlet RW. Computed tomography and blunt abdominal injury: patient selection based on examination, haematocrit and haematuria. Injury. 1997;28:181-5
33. Self ML, Dunn E. The benefit of routine thoracic, abdominal, and pelvic computed tomography to evaluate trauma patients with closed head injuries. Am J Surg. 2003;186:609-13.

34. Suthers SE, Tuggle DW, et al. Surgeon-directed ultrasound for trauma is a predictor of intra-abdominal injury in children. Am Surg. 2004;70:164-7.

35. Karom O, LaScala G, et al. Blunt abdominal trauma in children: a score to predict the absence of organ injury. J Pediatr. 2009;154:912-7.

36. Sauerland S, Neugebauer EA, et al. The reliability of clinical examination in detecting pelvic fractures in blunt trauma patients: a meta-analysis. Arch Orthop Trauma Surg. 2004;124:123-8.

37. Salvino CK, Gamelli RL, et al. Routine pelvic x-ray studies in awake blunt trauma patients: a sensible policy? J Trauma. 1992;33:413-6.

38. Gonzalez RP, Bukhalo M, et al. The utility of clinical examination in screening for pelvic fractures in blunt trauma. J Am Coll Surg. 2002;194:121-5.

39. Kirkpatrick AW, McKevitt E. Thoracolumbar spine fractures: is there a problem?". Can J Surg. 2002:45:21-4.

40. Terregino $C A$, Hughes $R$, et al. Selective indications for thoracic and lumbar radiography in blunt trauma. Ann Emerg Med. 1995;26:126-9.

41. Hsu JM, Ellis AM, et al. Thoracolumbar fracture in blunt trauma patients: guidelines for diagnosis and imaging. Injury. 2003;34:426-33.

42. Holmes JF, Mower WR, et al. Prospective evaluation of criteria for obtaining thoracolumbar radiographs in trauma patients. J Emerg Med. 2003;24:1-7.

43. Diaz JJ, Cullinane DC, EAST Practice Management Guideline Committee, et al. Practice management guidelines for the screening of thoracolumbar spine fracture. J Trauma. 2007;63:709-18.

44. Cooper $C$, Rodriguez $A$, et al. Falls and major injuries are risk factors for thoracolumbar fractures: cognitive impairment and multiple injuries impede the detection of back pain and tenderness. J Trauma. 1995;38:692-6.

45. Meldon SW, Moettus LN. Thoracolumbar spine fractures: clinical presentation and the effect of altered sensorium and major injury. J Trauma. 1995:39:1110-4

46. Inaba K, Demetriades D, et al. Clinical examination is insufficient to rule out thoracolumbar spine injuries. J Trauma. 2011;70:174-9.

47. Enderson BL, Maull KL. The tertiary trauma survey: a prospective study of missed injury. J Trauma. 1990;30:666-9.

48. Aaland MO, Smith K. Delayed diagnosis in a rural trauma center. Surgery. 1996:120:774-8

49. Born CT, DeLong WG, et al. Delayed identification of skeletal injury in multisystem trauma: the "missed" fracture. J Trauma. 1989;29:1643-6.

50. Laasonen EM, Kivioja A. Delayed diagnosis of extremity injuries in patients with multiple injuries. J Trauma. 1991;31:257-60.

51. Ward WG, Nunley JA. Occult orthopaedic trauma in the multiply injured patient. J Orthop Trauma. 1991;5:308-12.

52. Pfeifer R, Pape HC. Missed injuries in trauma patients: a literature review. Patient Saf Surg. 2008;23:2.

53. Ballas MT, Mannarino F. Commonly missed orthopedic problems. Am Fam Phys. 1998;15:267-74.

54. Corley FG. Examination and assessment of injuries and problems affecting the elbow, wrist, and hand. Emerg Med Clin North Am. 1984;2:295-312.

55. Darracq MA, Panacek EA, et al. Preservation of active range of motion after acute elbow trauma predicts absence of elbow fracture. Am J Emerg Med. 2007:26:779-82.

56. Lennon Rl, Alderson $\mathrm{G}$, et al. Can a normal range of elbow movement predict a normal elbow xray? Emerg Med J. 2007;24:86-8.

57. Baker WE, Wassermann J. Unsuspected vascular trauma: blunt arterial injuries. Emerg Med Clin North Am. 2004;22:1081-98.

58. Mosquera VX, Cuenca JJ, et al. Traumatic aortic injury score (TRAINS): an easy and simple score for early detection of traumatic aortic injuries in major trauma patients with associated blunt chest trauma. Intensive Care Med. 2012;38:1487-96.

59. Biffl WL, Moore FA, et al. Western Trauma Association critical decisions in trauma: Screening for and treatment of blunt cerebrovascular injuries. J Trauma. 2009:67:1150-3.

60. Burlew CC, Biffl WL, et al. Blunt cerebrovascular injuries: redefining screening critieria in the era of noninvasive diagnosis. J Trauma Acute Care Surg. 2012;72:330-5.

61. Bromberg WJ, Vogel TR, et al. Blunt cerebrovascular injury practice management guidelines: the Eastern association for the surgery of trauma. J Trauma. 2010;68:471-7. 